\title{
WEAK MORDELL-WEIL THEOREM
}

\author{
C. S. RAJAN
}

\begin{abstract}
We consider the weak Mordell-Weil theorem and introduce the notions of Selmer and Tate-Shafervich groups associated to an elliptic curve, and consider in brief the problem of computing the group $E(K) / m E(K)$.
\end{abstract}

\section{Mordell-Weil theorem}

Let $K$ be a number field and $E$ be an elliptic curve defined over $K$. The set of rational points $E(K)$ forms an abelian group. We have the fundamental theorem:

Theorem 1.1 (Mordell-Weil). $E(K)$ is a finitely generated abelian group.

It follows we can write $E(K)=\mathbb{Z}^{r} \oplus F$, where $r$ is the rank of $E(K)$ and $F$ is a finite torsion group. The question naturally arises of determining $F$ and the rank $r$ of $E(K)$. The theorems of Lutz-Nagell provide a satisfactory answer to the question of determining the torsion part $F$, given an equation of the curve. In this respect, much more is known regarding the size of the torsion groups that can occur for an elliptic curve defined over an arbitrary number field $K$. By the work of Mazur, Kamienny and Merel there exists a uniform upper bound for the torsion of elliptic curves defined over a number field $K$, depending only on the degree of $K$ over the rationals. Mazur has also classified the possible torsion subgroups of $E(\mathbb{Q})$ for elliptic curves defined over the rationals $\mathbb{Q}$.

It remains then to determine the rank of an elliptic curve $E(K)$ (if possible effectively). With this purpose, we examine the proof of the Mordell-Weil theorem, with the aim of making it effective. The proof can be divided into two parts. First we show the following;

Theorem 1.2 (Weak Mordell-Weil). For any positive integer $m$, the group $E(K) / m E(K)$ is finite.

The second part of the proof is to develop a theory of heights (we will not discuss this here). From the theory of heights of rational 
points of elliptic curves, it can be seen that if a finite set $A$ of elements of $E(K)$ can be found, such that they generate the group $E(K)$ modulo the subgroup $m E(K)$, then the finite set of elements of $E(K)$ with height at most the maximum of the heights of the elements in $A$, will generate $E(K)$. Thus the problem of computing the rank of the Mordell-Weil group, is reduced to the problem of computing the generators of $E(K) / m E(K)$. With this purpose in mind, we look at the proof of the weak Mordell-Weil theorem.

\section{Galois COHomology}

Over an algebraic closure $\bar{K}$ of $K$, the multiplication map $m$ : $E(\bar{K}) \rightarrow E(\bar{K})$ is surjective. For instance over the complex numbers, it follows by uniformization that $E(\mathbb{C}) \simeq \mathbb{C} /(\mathbb{Z}+\mathbb{Z} \tau)$ for some $\tau$ with $\operatorname{Im}(\tau) \neq 0$, and it can be checked that multiplication by $m$ is surjective. However over a number field $K$, the solutions to an equation of the form $m Q=P$, for some $P \in E(K)$, lie in $E(\bar{K})$. The problem then is to control the field extensions of $K$ generated by $Q$ as $P$ varies over the rational points of $E$ over $K$, and this is the main theme of the proof. A convenient way to do this is via Galois cohomology.

Let $G$ be a (finite) group, and $M$ be a $G$-module, i.e. an abelian group $M$ with an action of $G: M \times G \rightarrow M$, denoted by $(x, \sigma) \mapsto x^{\sigma}$. Given such data, we have the cohomology groups $H^{i}(G, M)$ for $i \geq 0$, satisfying:

a) The zeroth cohomology is the space of invariants of $M$ with respect to the action of $G$ :

$$
H^{0}(G, M)=M^{G}=\left\{x \in M \mid x^{\sigma}=x, \forall \sigma \in G\right\} .
$$

b) Given a short exact sequence of $G$-modules,

$$
0 \rightarrow M^{\prime} \rightarrow M \rightarrow M^{\prime \prime} \rightarrow 0,
$$

there is an associated long exact sequence of cohomology groups

$$
\cdots \rightarrow H^{i-1}\left(G, M^{\prime \prime}\right) \rightarrow H^{i}\left(G, M^{\prime}\right) \rightarrow H^{i}(G, M) \rightarrow H^{i}\left(G \cdot M^{\prime \prime}\right) \rightarrow \cdots .
$$

Explicitly, $H^{1}(G, M)$ can be described as follows: Let

$$
Z^{1}(G, M)=\left\{\xi: G \rightarrow M \mid \xi(\sigma \tau)=\xi(\sigma)^{\tau}+\xi(\tau), \forall \sigma, \tau \in G\right\},
$$

be the groups of cocycles.

$$
B^{1}(G, M)=\left\{\xi: G \rightarrow M \mid \xi(\sigma)=a^{\sigma}-a, \text { for some } a \in G\right\},
$$

be the group of coboundaries. Then

$$
H^{1}(G, M)=Z^{1}(G, M) / B^{1}(G, M) .
$$

Example 2.1. If $G$ acts trivially on $M$, then $H^{1}(G, M)=\operatorname{Hom}(G, M)$. 
The absolute Galois group of $K$ is defined as,

$$
G_{K}:=\lim _{\{L: K \subset L \subset \bar{K}\}} \operatorname{Gal}(L / K),
$$

given as a projective limit of the finite Galois groups $\operatorname{Gal}(L / K)$, where $L$ runs over the finite Galois extensions of $K$ contained inside $\bar{K}$. If $L$ is a finite Galois extension of $K$ with Galois group $\operatorname{Gal}(L / K)$ then using the surjective homomorphism $G_{K} \rightarrow \operatorname{Gal}(L / K)$ we can treat any $\operatorname{Gal}(L / K)$ module $M_{L}$ as a $G_{K}$ module. More generally $M=\cup_{L} M_{L}$, where $M_{L}$ is a $\operatorname{Gal}(L / K)$-module and $L$ runs over all finite Galois extensions of $K$ contained inside $\bar{K}$ and the actions of $\operatorname{Gal}(L / K)$-are compatible, then such an $M$ is called a $G_{K}$-module. For such an $M$ we define the Galois cohomology groups

$$
H^{i}\left(G_{K}, M\right)=\underline{\lim }_{\{L: K \subset L \subset \bar{K}\}} H^{i}\left(\operatorname{Gal}(L / K), M_{L} .\right)
$$

Alternatively it is possible to define the cohomology groups working with continuous cochains, where $G_{K}$ is given the profinite topology and $M$ is considered as a discrete $G_{K}$-module.

Suppose $X$ is a 'geometric object' defined over $K$, and consider the group $\operatorname{Aut}(X)$ of automorphisms of $X$. Even though $\operatorname{Aut}(X)$ is nonabelian, it is possible to define a first cohomology set $H^{1}\left(G_{K}, \operatorname{Aut}(X)\right)$, in a similar manner as was done for abelian coefficients (if $A \subset \operatorname{Aut}(X)$ is an abelian subgroup then the set $H^{1}\left(G_{K}, A\right)$ has a natural group structure). In this case, there is a heuristic giving a geometric intrepretation the Galois cohomology sets $H^{1}\left(G_{K}\right.$, Aut $\left.(X)\right)$. The set $H^{1}\left(G_{K}, \operatorname{Aut}(X)\right)$ parametrizes the space of ' $K$-forms of $X$ ', where by a $K$-form of $X$ we mean an object $Y$ defined over $K$, together with an isomorphism $\phi: Y \rightarrow X$ defined over $\bar{K}$. Attached to such a $K$-form the corresponding 'Galois cocycle' $\xi_{\phi} \in H^{1}\left(G_{K}, \operatorname{Aut}(X)\right)$ is defined by

$$
\xi_{\phi}(\sigma)=\phi^{\sigma} \circ \phi^{-1} \text {. }
$$

2.1. Kummer sequence. For a positive integer $m$, let

$$
E[m]=\operatorname{Ker}(m: E(\bar{K}) \rightarrow E(\bar{K})),
$$

be the group of $m$-torsion points of $E$. We have the following exact sequence of $G_{K}$-modules, the Kummer sequence:

$$
0 \rightarrow E[m] \rightarrow E(\bar{K}) \stackrel{m}{\rightarrow} E(\bar{K}) \rightarrow 0 .
$$

The associated long exact sequence of Galois cohomology groups is

$$
\begin{aligned}
0 \rightarrow E(K)[m] & \rightarrow E(K) \stackrel{m}{\rightarrow} E(K) \stackrel{\delta}{\rightarrow} H^{1}\left(G_{K}, E[m]\right) \\
& \rightarrow H^{1}\left(G_{K}, E(\bar{K}) \stackrel{m}{\rightarrow} H^{1}\left(G_{K}, E(\bar{K})\right) .\right.
\end{aligned}
$$


The connecting morphism $\delta$ is given as follows: let $P \in E(K)$. Since the multiplication by $m$ map is surjective, we can find $Q \in E(\bar{K})$ such that $m Q=P$. Then we see that the element $\delta(P) \in H^{1}\left(G_{K}, E[m]\right)$ is the class of the cocycle

$$
\delta(P)(\sigma)=Q^{\sigma}-Q, \quad \sigma \in G_{K} .
$$

From the long exact sequence above, we obtain the fundamental exact sequence,

$$
0 \rightarrow E(K) / m E(K) \stackrel{\delta}{\rightarrow} H^{1}\left(G_{K}, E[m]\right) \stackrel{j}{\rightarrow} H^{1}(K, E)[m] \rightarrow 0
$$

where we write $H^{1}(K, E)$ for $H^{1}\left(G_{K}, E(\bar{K})\right)$ and

$$
H^{1}(K, E)[m]=\operatorname{Ker}\left(H^{1}\left(G_{K}, E(\bar{K})\right) \stackrel{m}{\rightarrow} H^{1}\left(G_{K}, E(\bar{K})\right)\right) .
$$

\section{Proof of Weak Mordell-Weil}

We now proceed to the proof of the weak Mordell-Weil theorem. First we do a preliminary reduction to the case when $E[m] \subset E(K)$.

Let $L$ be a finite normal extension of $K$ such that $E[m] \subset E(L)$, and assume now that $E(L) / m E(L)$ is finite. We would like to conclude that $E(K) / m E(K)$ is finite. From the inclusion

$$
E(K) /(m E(L) \cap E(K)) \subset E(L) / m E(L),
$$

given by $K \subset L$, it follows that $E(K) /(m E(L) \cap E(K))$ is finite.

Now if $P \in m E(L) \cap E(K)$, then we can find $Q \in E(L)$ satisfying $m Q=P$. From the description of the connecting morphism given above, it follows that the cohomology class $\delta(P) \in H^{1}\left(G_{K}, E[m]\right)$ restricts to the trivial cohomology classes in $H^{1}\left(G_{L}, E[m]\right)$. Recall that we have the inflation-restriction exact sequence,

$$
H^{1}\left(G_{L / K}, E[m]\right) \rightarrow H^{1}\left(G_{K}, E[m]\right) \rightarrow H^{1}\left(G_{L}, E[m]\right) .
$$

Hence we obtain an inclusion

$$
(m E(L) \cap E(K)) / m E(K) \subset H^{1}\left(G_{L / K}, E[m]\right) .
$$

But $H^{1}\left(G_{L / K}, E[m]\right)$ can be seen to be finite, being the cohomology of a finite group with values in a finite module. Hence we conclude that if $E(L) / m E(L)$ is finite, so is $E(K) / m E(K)$.

Hence we can assume that $E[m] \subset E(K)$. In particular $G_{K}$ acts trivially on $E[m]$ and so,

$$
H^{1}\left(G_{K}, E[m]\right)=\operatorname{Hom}_{c t}\left(G_{K}, E[m]\right):=\underset{L: K \subset L \subset \bar{K}}{\lim _{\longrightarrow}} \operatorname{Hom}(G(L / K), E[m]) .
$$

We have the exact sequence

$$
0 \rightarrow E(K) / m E(K) \rightarrow \operatorname{Hom}_{c t}\left(G_{K}, E[m]\right) \stackrel{j}{\rightarrow} H^{1}(K, E)[m] \rightarrow 0 .
$$


To conclude the proof of the weak Mordell-Weil theorem, we have to show that the image of $E(K) / m E(K)$ inside $\operatorname{Hom}_{c t}\left(G_{K}, E[m]\right)$ is finite. Let $S$ be a finite set of places of $K$ containing the places of bad reductions of $E$, the places dividing $m$, and the archimedean places of $K$. It can be seen that the homomorphism $\delta(P) \in \operatorname{Hom}\left(G_{K}, E[m]\right)$ corresponding to a point $P \in E(K) / m E(K)$ is trivial restricted to the inertia subgroups for a place $v \notin S$ (this says that the cohomology class is unramified at such places). Consequently the homomorphism $\delta_{P}$ factors via the quotient group $G_{K, S}$ of $G_{K}$, where $G_{K, S}$ is the Galois group of the maximal abelian extension of $K$ contained in $\bar{K}$ and unramified outside $S$. It is a consequence of Minkowski's theorem that $G_{K, S}$ is a finitely generated as a topological group. Since $G_{K, S}$ is topologically finitely generated, and $E[m]$ is a finite group, it follows that $\operatorname{Hom}_{c t}\left(G_{K}, E[m]\right)$ is finite, and this completes the proof of the weak Mordell-Weil theorem.

From the Kummer sequence we have an embedding of $E(K) / m E(K)$ into the finite cohomology module $H^{1}\left(G_{K}, E[m]\right)$. Given $E$, in principle it is possible to compute $E[m]$ and the cohomology module $H^{1}\left(G_{K}, E[m]\right)$. From the exactness of the fundamental sequence, it follows that the image of $E(K) / m E(K)$ consists of those cohomology classes $\xi \in H^{1}\left(G_{K}, E[m]\right)$ such that the corresponding element $j(\xi) \in H^{1}(K, E)$ is trivial. We now examine the geometric interpretation of the group $H^{1}(K, E)$, which will lead us to apply the local-global principle in this situation.

\section{Principal Homogeneous spaces}

We first consider the cohomology groups $H^{1}(K, E)$ from the viewpoint of the geometric heuristic outlined above.

Definition 4.1. A principal homogeneous space for $E$ over $K$ is a pair $(C, \mu)$ consisting of a smooth projective curve $C$ over $K$, and a morphism $\mu: C \times E \rightarrow C$ defined over $K$ satisfying the following:

(1) $\left.\mu\right|_{C \times O}: C \times\{O\} \rightarrow C$ is the identity morphism where $O$ is the identity element of $E$.

(2) Let $a: E \times E \rightarrow E$ denote the addition map on the elliptic curve. The following diagram is commutative:

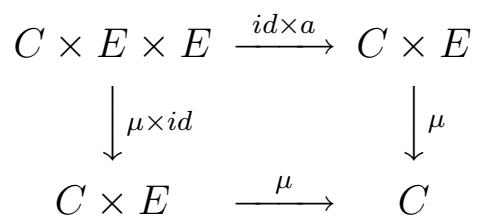

(3) The map $C \times E \rightarrow C \times C$ given by $(i d, \mu)$ is an isomorphism. 
In terms of points with values in $\bar{K}$, the axioms for a principal homogeneous space can be formulated as:

- $\mu(p, O)=p$ for a point $p$ of $C$.

- for points $p$ of $C$ and $P, Q$ points of $E$, we have $\mu(\mu(p, P), Q)=$ $\mu(p, P+Q)$.

- for any points $p, q$ of $C$, there exists a unique point $P$ of $E$ such that $\mu(p, P)=q$.

In particular we obtain a map $\nu: C(\bar{K}) \times C(\bar{K}) \rightarrow E(\bar{K})$ which maps a pair $(p, q)$ to the unique point $P$. We will also denote $\nu(p, q)$ by $q-p$.

Example 4.1. Via the addition map $a: E \times E \rightarrow E, E$ becomes a principal homogeneous space for $E$ over $K$. We will refer to this as the trivial principal homogeneous space of $E$ over $K$.

Definition 4.2. Two principal homogeneous space's $(C, \mu)$ and $\left(C^{\prime}, \mu^{\prime}\right)$ for $E$ over $K$ are equivalent, if there is an isomorphism $\theta: C \rightarrow C^{\prime}$ defined over $K$, such that the following diagram is commutative:

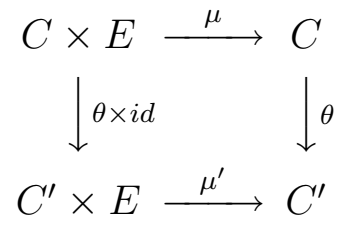

The Weil-Chatelet set $W C(E / K)$ is the collection of equivalence classes of principal homogeneous space for $E$ over $K$. Suppose now that $(C, \mu)$ is a principal homogeneous space for $E$ over $K$ and that $C(K)$ is non-empty. Choose a point $p_{0} \in C(K)$. Identify $E$ with the fibre of $C \times E$ over the point $p_{0} \in C(K)$, and define a morphism $\theta: E \rightarrow C$ by,

$$
\theta=\left.\mu\right|_{p_{0} \times E}: E \rightarrow C .
$$

Proposition 4.1. $\theta$ is an isomorphism and gives an equivalence of $C$ to the trivial principal homogeneous space defined in Example 4.1.

In particular it follows that $E$ is isomorphic to $C$ over $\bar{K}$, i.e., $C$ is a form of $E$ over $K$. We will leave the proof of this proposition to the reader and refer to the bibliography for further details (and for what follows).

\section{Theorem 4.2.}

$$
W C(E / K) \simeq H^{1}(K, E) .
$$

This is the geometric interpretation of the cohomology groups that we had hinted at before. The trivial cohomology class corresponds to the trivial principal homogeneous space defined by $E$. Since $E \subset$ 
Aut(E) as the subgroup of translations via this isomorphism $W C(E / K)$ acquires the structure of an abelian group (it is possible to define the group structure directly without the aid of cohomology, and then check that indeed the isomorphism of sets provided by the above theorem is an isomorphism of groups).

Proof. We give a brief outline of the proof, and for details refer to $[S]$. Given a representative $(C, \mu)$ of a class in $W C(E / K)$, choose a point $p_{0} \in C(\bar{K})$, and send it to the cohomology class defined by

$$
\xi_{C}(\sigma)=p_{0}^{\sigma}-p_{0},
$$

for an element $\sigma \in G_{K}$. It is easy to check that this gives a well-defined cohomology class and that the map is injective. The essential point is to prove the surjectivity. Over $\bar{K}$ the function field of $C$ is isomorphic to the function field $\mathcal{F}$ of $E$. Suppose $C$ is a $K$-form of $E$ with an isomorphism $\phi: C \rightarrow E$ over $\bar{K}$. Given a rational function $f \in \mathcal{F}$, we have

$$
(f \circ \phi)^{\sigma}=\left(f^{\sigma} \circ\left(\phi^{\sigma} \circ \phi^{-1}\right)\right) \circ \phi .
$$

The above calculation provides us with the clue to define a new action of $G_{K}$ on $\mathcal{F}$ associated to a co-cycle $\xi \in H^{1}\left(G_{K}, E\right)$ :

$$
f^{N(\sigma)}=f^{\sigma} \circ\left(-\xi_{\sigma}\right) .
$$

Here $\sigma \in G_{K}, f \in \mathcal{F}$ and by $f^{\sigma}$ (resp. $f^{N(\sigma)}$ )we denote the action (resp. new action) of $G_{K}$ on $\mathcal{F}$. Let

$$
\mathcal{F}_{\xi}=\left\{f \in \mathcal{F} \mid f^{\hat{\sigma}}=f\right\} .
$$

To conclude that $\mathcal{F}_{\xi}$ is the function field of a curve $C$ defined over $K$, we need to check that i) $\mathcal{F}_{\xi} \cap \bar{K}=K$ and ii) $\bar{K} \mathcal{F}_{\xi}=\mathcal{F}$.

This is achieved by an application of Hilbert Theorem 90 to obtain the following descent lemma:

Lemma 4.1. Let $V$ be $\bar{K}$-vector space, such that $V=\cup_{L} V^{G_{L}}$, where $L$ runs over the finite extensions of $K$. Then

$$
V \simeq \bar{K} \otimes V^{G_{K}}
$$

Proof. Let $L$ be a finite Galois extension of $K$, and let $v \in V^{G_{L}}$. Let $\alpha_{1}, \cdots, \alpha_{d}$ be a basis for $L / K$ and $\sigma_{1}, \cdots, \sigma_{d}$ be the elements of the Galois group of $L$ over $K$. Then

$$
\operatorname{Tr}_{L / K}\left(\alpha_{i} v\right)=\sum_{j=1}^{d}\left(\alpha_{i} v\right)^{\sigma_{j}} \in V^{G_{K}} .
$$

The lemma follows from the fact that the $d \times d$-matrix $\left(\alpha_{i}^{\sigma_{j}}\right)$ is invertible. 
From this we conclude that $\mathcal{F}_{\xi}$ has transcendence degree one over $K$, and thus there exists a curve $C_{\xi}$ defined over $K$ together with an isomorphism $\phi: C_{\xi} \rightarrow E$ defined over $\bar{K}$. From the construction, it can be checked that $C_{\xi}$ is a principal homogeneous space space for $E$, and that the corresponding cocyle is $\xi$ (this is the reason for the minus sign in the definition of the Galois action).

\section{Selmer and Tate-Shafarevich groups; applications of THE LOCAL-GLOBAL PRINCIPLE}

It follows from the fundamental exact sequence 2.2, that the image of $E(K) / m E(K)$ consists of those cohomology classes $\xi \in H^{1}\left(G_{K}, E[m]\right)$ such that the corresponding principal homogeneous space $C_{\xi}$ determined by $\pi(\xi) \in H^{1}(K, E)$ is trivial, i.e., $C_{\xi}(K)$ is non-empty. Thus we are reduced to the problem of determining the existence of a rational point on a genus 1 curve $C_{\xi}$ defined over a number field.

The standard approach to such a problem is the local-global principle: consider the corresponding question over each of the local completions $K_{v}$ of $K$ at a place $v$ of $K$. At a non-archimedean place where the curve has good reduction, this problem reduces by Hensel's lemma to the problem of finding rational points of a given curve over finite fields. (over $\mathbb{R}$, the problem is easy to solve). For each place $v$ of $K$, choose an extension of $v$ to $\bar{K}$. We have a commutative diagram

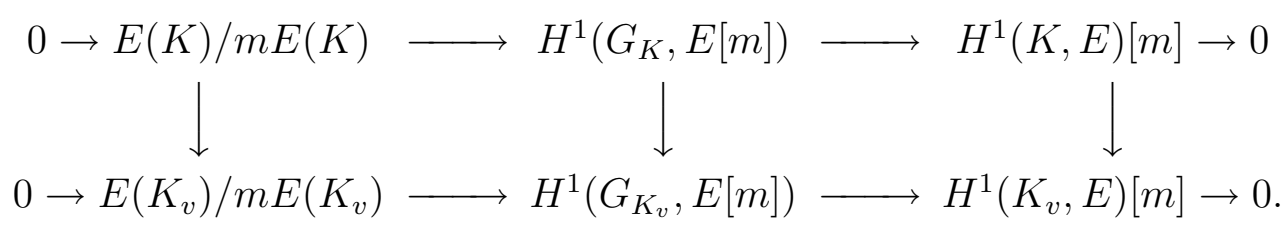

Definition 5.1. The Selmer group of exponent $m$ is,

$$
S^{(m)}(E / K)=\operatorname{Ker}\left(H^{1}\left(G_{K}, E[m]\right) \rightarrow \prod_{v} W C\left(E / K_{v}\right)\right)
$$

where the product runs over all the places $v$ of $K$. The Tate-Shafarevich group is defined to be the 'obstruction' to the Hasse principle,

$$
\operatorname{III}(E / K)=\operatorname{Ker}\left(W C(E / K) \rightarrow \prod_{v} W C\left(E / K_{v}\right)\right) .
$$

In other words, the elements of the Tate-Shafarevich group consists of those principal homogoneous spaces $C$ for $E$ over $K$, which are locally trivial at all places $v$ of $K$ i.e., $C\left(K_{v}\right)$ is non-empty for each place $v$ of $K$. We have the exact sequence

$$
0 \rightarrow E(K) / m E(K) \rightarrow S^{(m)}(E / K) \rightarrow \operatorname{III}(E / K)[m] \rightarrow 0 .
$$


The weak Mordell-Weil theorem can be recast as saying,

Theorem 5.1. $S^{(m)}(E / K)$ is finite.

To see this, we have to just observe that $S^{(m)}(E / K) \subset H^{1}\left(G_{K, S}, E[m]\right)$ following the proof of weak Mordell-Weil given above.

Remark 5.1. To check whether an element $\xi \in H^{1}\left(G_{K, S}, E[m]\right)$ belongs to $S^{(m)}(E / K)$ it is enough to check that $C_{j_{v}(\xi)}\left(K_{v}\right)$ is non-empty for $v \in S$, where $j_{v}: H^{1}\left(G_{K, S}, E[m]\right) \rightarrow H^{1}\left(K_{v} E\right)$ is the natural map. This follows from Lang's theorem, that any principal homogeneous space of an algebraic group $G$ over a finite field is trivial, and then by applying Hensel's lemma. Thus it is enough to check at only finitely many places whether the associated principal homogeneous space has a rational point over the corresponding completion.

The basic conjecture is the following finiteness conjecture:

Conjecture 5.1. $\operatorname{III}(E / K)$ is finite.

To see how this conjecture can be used to obtain generators of $E(K) / m E(K)$, define

$$
S^{(m, n)}(E / K)=\operatorname{Image}\left(S^{\left(m^{n}\right)}(E / K) \stackrel{m^{n-1}}{\longrightarrow} S^{(m)}(E / K)\right) .
$$

We have an exact sequence

$$
0 \rightarrow E(K) / m E(K) \rightarrow S^{(m, n)}(E / K) \rightarrow m^{n-1} \operatorname{III}(E / K)\left[m^{n}\right] \rightarrow 0 .
$$

For any positive real number $r$, let $T_{(m, r)}(E / K)$ be the subgroup of $S^{(m)}(E / K)$ generated by the images under the connecting morphism $\delta$ of points of $E(K)$ of height less than $r$. We have an inclusion $T_{(m, r)}(E / K) \subset S^{(m, n)}(E / K)$. If $\operatorname{III}(E / K)$ is finite, then we have for some $m, r, n$ that $T_{(m, r)}(E / K)=S^{(m, n)}(E / K)$, and this allows us to find generators for $E(K) / m E(K)$, since the groups $T_{(m, r)}(E / K)$ and $S^{(m, n)}(E / K)$ are computable.

Remark 5.2. For the method to be effective, it remains to give a suitable upper bound for the size of $\operatorname{III}(E / K)$. Depending on some standard conjectures, it is possible to obtain bounds of the form

$$
|\operatorname{III}(E / K)|=O\left(N^{1 / 2+\epsilon}\right),
$$

for any $\epsilon$ positive, where the implicit constant depends only on $\epsilon$ and $K$, and $N$ is the norm of the conductor of the elliptic curve. Assuming the finiteness of $\operatorname{III}(E / K)$, such estimates are known over function fields, i.e., when $K$ is the function field of a curve over a finite field. 


\section{REFERENCES}

[C] J. W. S. Cassels, Diophantine equations with special reference to elliptic curves, J. London Math. Soc. 41 (1966) 193-291.

[S] J. Silverman, The Arithmetic of elliptic curves, Graduate Texts in Mathematics, 106 Springer-Verlag.

Tata Institute of Fundamental Research, Homi Bhabha Road, BomBAY - 400 005, INDIA.

E-mail address: rajan@math.tifr.res.in 